\title{
Simplifying Non-Simple Fan-Planar Drawings ${ }^{\star}$
}

\author{
Boris Klemz ${ }^{1[0000-0002-4532-3765]}$, Kristin Knorr ${ }^{2[0000-0003-4239-424 X]}$, \\ Meghana M. Reddy ${ }^{3[0000-0001-9185-1246]}$, and Felix \\ Schröder $4[0000-0001-8563-3517]$ \\ ${ }^{1}$ Institut für Informatik, Universität Würzburg, Würzburg, Germany \\ boris.klemz@uni-wuerzburg.de \\ ${ }^{2}$ Institut für Informatik, Freie Universität Berlin, Berlin, Germany \\ knorrkri@inf .fu-berlin.de \\ 3 Department of Computer Science, ETH, Zürich, Switzerland \\ meghana.mreddy@inf .ethz.ch \\ 4 Institut für Mathematik, Technische Universität Berlin, Berlin, Germany \\ fschroed@math.tu-berlin.de
}

\begin{abstract}
A drawing of a graph is fan-planar if the edges intersecting a common edge $a$ share a vertex $A$ on the same side of $a$. More precisely, orienting $e$ arbitrarily and the other edges towards $A$ results in a consistent orientation of the crossings. So far, fan-planar drawings have only been considered in the context of simple drawings, where any two edges share at most one point, including endpoints. We show that every non-simple fan-planar drawing can be redrawn as a simple fan-planar drawing of the same graph while not introducing additional crossings. Combined with previous results on fan-planar drawings, this yields that $n$-vertex-graphs having such a drawing can have at most $6.5 n$ edges and that the recognition of such graphs is NP-hard. We thereby answer an open problem posed by Kaufmann and Ueckerdt in 2014.
\end{abstract}

Keywords: Simple topological graphs · Fan-planar graphs · Beyondplanar graphs · Graph drawing.

\section{Introduction}

In a fan-planar drawing of a graph, each edge $a$ is either not involved in any crossing or its crossing edges $c_{1}, \ldots, c_{k}$ have a common endpoint $A$ that is on a common side of $a$, i.e., orienting $a$ arbitrarily and the edges $c_{1}, \ldots, c_{k}$ towards $A$

\footnotetext{
* This work was initiated at the $5^{\text {th }}$ DACH Workshop on Arrangements and Drawings, which was conducted online, via gathertown, in March 2021. The authors thank the organizers of the workshop for inviting us and providing a productive working atmosphere. B. K. was partially supported by DFG project WO 758/11-1. M. M. R. is supported by the Swiss National Science Foundation within the collaborative DACH project Arrangements and Drawings as SNSF Project 200021E-171681. K. K. is supported by DFG Project MU 3501/3-1 and within the Research Training Group GRK 2434 Facets of Complexity. F. S. is supported by the German Research Foundation DFG Project FE 340/12-1.
} 
results in a consistent orientation of the crossings on $a$ (either $a$ crosses each $c_{i}$ from left to right at each crossing, or it crosses each $c_{i}$ from right to left at each crossing); for illustrations refer to Figure 1. We call $A$ the special vertex of $a$. All graphs in this paper are simple, that is, we do not allow parallel edges or self-loops. Hence, the vertex $A$ is uniquely defined if $k \geq 2$. If $k=1$, then $A$ is an arbitrary endpoint of $c_{1}$.

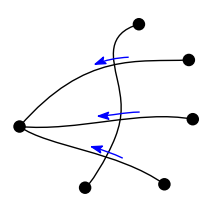

(a)

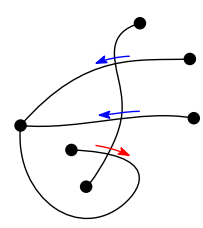

(b)

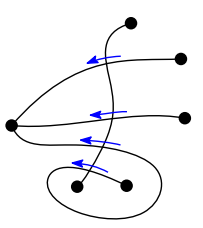

(c)

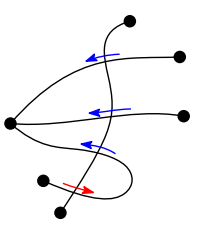

(d)

Fig. 1. Drawings that are (a) simple and fan-planar, (b) simple and not fan-planar, (c) non-simple and fan-planar, and (d) non-simple and not fan-planar.

Previous literature is exclusively concerned with fan-planar drawings that are also simple, meaning that each pair of edges intersects in at most one point, which can be either an endpoint or a proper crossing. Simple drawings can be characterized in terms of two forbidden crossing configuration 5 (see Figure 2):

S1 Two adjacent edges cross.

S2 Two edges cross at least twice.

Simple drawings that are fan-planar can be characterized in terms of two additional forbidden crossing configurations [17] (see Figure 2):

SF1 Two independent edges cross a common third edge.

SF2 Two adjacent edges cross a third edge $a$ such that their common endpoint $A$ is not on a common side of $a$.

In this paper, we study non-simple fan-planar drawings and how to turn them into simple fan-planar drawings.

Previous and related work. A drawing is $k$-planar if each edge is crossed at most $k$ times and a graph is $k$-planar, if it admits such a drawing [19. A $k$-quasiplanar graph can be drawn such that no $k$ edges mutually cross - such a drawing is called $k$-quasiplanar 2. Kaufmann and Ueckerdt [17] introduced the notion of fan-planarity in 2014. They describe the class of graphs representable by simple fan-planar drawing: $\AA^{6}$ as somewhere between 1-planar graphs and 3-quasiplanar

${ }^{5}$ In the literature, usually more obstructions are mentioned, which we exclude for all drawings (simple or not), see Section 2

${ }^{6}$ In [17, these graphs are called fan-planar. We do not use this terminology to avoid mix-ups with the class of graphs admitting (not necessarily simple) fan-planar drawings. 


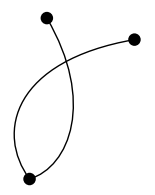

(a) S1

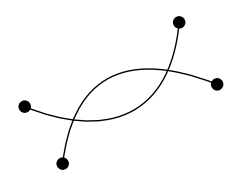

(b) S2

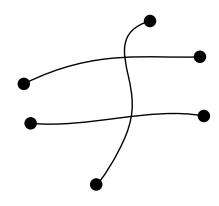

(c) SF1

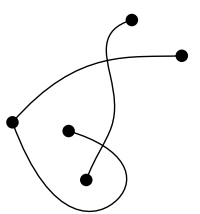

(d) SF2

Fig. 2. Forbidden configurations in simple fan-planar drawings.

graphs. Indeed, every 1-planar graph admits a simple 1-planar drawing. Since such a drawing cannot contain configuration SF1 or SF2, it is fan-planar. Moreover, a simple fan-planar drawing cannot contain three mutually crossing edges and, therefore, it is 3-quasiplanar. Binucci et al. 9] have shown that for each $k \geq 2$ the class of graphs admitting simple $k$-planar drawings and the class of graphs admitting simple fan-planar drawings are incomparable. In contrast, every so-called optimal 2-planar graph admits a simple fan-planar drawing 7 . This follows from the fact that these graphs can be characterized as the graphs obtained by drawing a pentagram in the interior of each face of a pentagulation [7, which yields a fan-planar drawing. Angelini et al. [3] introduced a drawing style that combines fan-planarity with a visualization technique called edge bundling [1415|20. Each of their so-called 1-sided 1-fan-bundle-planar drawings represents a graph that is also realizable as a simple fan-planar drawing, but the converse is not true [3]. Brandenburg [10] examines fan-crossing drawings, where all edges crossing a common edge share a common endpoint (in particular, this implies that SF1 is forbidden), as well as adjacency-crossing drawings, where $\mathrm{SF} 1$ is the only obstruction. Simple fan-planar drawings are somewhat opposite to simple $k$-fan-crossing-free [11] drawings, where no $k \geq 2$ adjacent edges cross another common edge.

The maximum number of edges in a simple fan-planar drawing on $n$ vertices is upperbounded by $6.5 n-20$ [17, which follows from the known density bounds for 3-quasiplanar graphs [1]. A better upper bound of $5 n-10$ edges was claimed in a preprint [17]. However, the corresponding proof appears to be flawed. We spoke with the authors and they confirmed that the current version of their proof is not correct and that they do not see a simple way to fix it7? Kaufmann and Ueckerdt [17] described an infinite family of simple fan-planar drawings

\footnotetext{
${ }^{7}$ More specifically, the statement and proof of [17, Lemma 1] are incorrect. A counterexample can be obtained by removing the edge $g$ from the construction illustrated in Figure 8 (vertices $R, B$ correspond to the vertices $u, w$ in [17. Lemma 1]); for a formal description of the construction see Lemma 3

After our submission to GD'21, the authors of 17 have uploaded a new version 18 of their preprint in which they state a different definition of fan-planarity with an additional forbidden crossing configuration; also see [18, last paragraph of Section 1].
} 
with $5 n-10$ edges. The same lower bound also follows from the aforementioned connection to optimal 2-planar graphs [7].

The recognition of graphs realizable as simple fan-planar drawings is NPhard [9]. The same statement also holds in the fixed rotation system setting [5], where the cyclic order of edges incident to each vertex is prescribed as part of the input. Consequently, efficient algorithms have only been discovered for special graph classes [5] and for restricted drawing styles [5]8].

For a more comprehensive overview of previous work related to fan-planarity, we refer to a very recent survey article dedicated to fan-planarity due to Bekos and Grilli $[6$. The study of fan-planarity also falls in line with the recent trend of studying so-called beyond-planar graph classes, whose corresponding drawing styles permit crossings in restricted ways only. Apart from $k$-planar [19], $k$ quasiplanar [2], $k$-fan-crossing-free [11, fan-bundle-planar [3], fan-crossing [10, adjacency-crossing [17, and fan-planar [17] drawings, which have already been mentioned above, several other classes of beyond-planar graphs and their corresponding drawing styles have been studied, e.g.: $k$-gap-planar drawings [4] (each crossing is assigned to one of the involved edges such that each edge is assigned at most $k$ crossings), RAC-drawings 12 (straight-line drawings with right angle crossings), and many more. We refer to [13|16] for recent surveys on beyond-planar graphs.

Contribution. A fan-planar drawing that is not simple may contain configuration S1. Configuration S2 is allowed in a partial sense: two edges may cross any number of times, but only if orienting them arbitrarily results in a consistent orientation of their crossings; cf. Figures 1(c) and (d). Recall that every simple fan-planar drawing is 3-quasiplanar. In contrast, Figure 3(a) depicts a non-simple fan-planar drawing that is not 3-quasiplanar, which suggests that graphs admitting non-simple fan-planar drawings are not necessarily 3-quasiplanar. Consequently, the density bound of $6.5 n-20$ [1] for 3-quasiplanar graphs does not directly carry over. However, the depicted graph is just a $K_{3}$, which can obviously be redrawn as a simple (fan-)planar drawing. This raises two very natural questions:

1. Is the largest number of edges in a $n$-vertex non-simple fan-planar drawing larger than the number of edges in any $n$-vertex simple fan-planar drawing?

2. Which non-simple fan-planar drawings can be redrawn as simple fan-planar drawings of the same graph?

Question 1 is also mentioned as an open problem by Kaufmann and Ueckerdt [17. Regarding question 2, we remark that the standard method for simplifying the configurations S1 and S2 does not necessarily maintain fan-planarity, see Figures 3(b) and (c). As our main result, we answer both questions, thereby solving the open problem by Kaufmann and Ueckerdt:

Theorem 1. Every non-simple fan-planar drawing can be redrawn as a simple fan-planar drawing of the same graph without introducing additional crossings. 


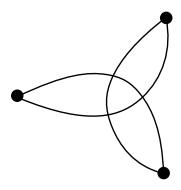

(a)

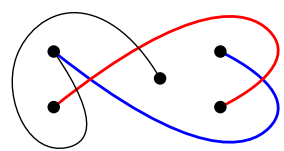

(b)

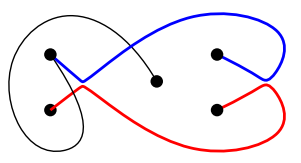

(c)

Fig. 3. (a) A 3-quasiplanar non-simple fan-planar drawing. (b) A non-simple fan-planar drawing. Applying the standard procedure for simplifying configuration S2 yields the drawing in (c), which is not fan-planar since the black edge crosses two independent edges.

The proof of Theorem 1 is constructive and gives rise to an efficient algorithm for simplifying a given fan-planar drawing. Combined with the aforementioned previous results regarding the density [117] and the recognition complexity [9] of graphs realizable as simple fan-planar drawings, we obtain:

Corollary 1. Every (not necessarily simple) fan-planar drawing realizes a 3quasiplanar graph.

Corollary 2. Every (not necessarily simple) fan-planar drawing on $n$ vertices has at most $6.5 n-20$ edges.

Corollary 3. Recognizing graphs that admit (not necessarily simple) fan-planar drawings is NP-hard.

We start with some basic terminology and conventions in Section 2, The algorithm for simplifying non-simple fan-planar drawings is described in Section 3 .

\section{Terminology}

In all drawings in this paper, edges are represented by simple curves. We assume no two edges touch, that is, meet tangentially. Further, we assume that no three edges share a common crossing and that edges do not contain vertices except their endpoints. Let $\Gamma$ be a drawing of a graph $G$. A redrawing of $\Gamma$ is a drawing of $G$. Redrawing an edge $e$ in $\Gamma$ refers to the process of obtaining a redrawing $\Gamma^{\prime}$ of $\Gamma$ such that $(\Gamma-e)=\left(\Gamma^{\prime}-e\right)$.

In the beginning of Section 1, we introduced the notion of special vertices for crossed edges. To streamline the arguments, we also assign an arbitrarily chosen special vertex to each uncrossed edge. Let $e$ and $f$ be edges that cross and let $E$ be the special vertex of $e$. We define the $i^{\text {th }}$ crossing of $f$ with $e$ as the $i^{\text {th }}$ crossing between $f$ and $e$ encountered when traversing $f$ from endpoint $E$. For example, in Figure 5(a), the first crossing of $g$ with $b$ is $x$ and the second crossing is $y$. 


\section{The Redrawing Procedure}

We prove Theorem 1 by providing an algorithm that redraws the edges of a non-simple fan-planar drawing $\Gamma$ to obtain a simple fan-planar drawing. It is based on three subroutines (Lemmata 1, 2 and 4), which can be iteratively applied to remove crossings between adjacent edges (configuration S1) and multiple crossings between pairs of edges (configuration S2). More specifically, the first procedure (Lemma 1) eliminates a particular type of adjacent crossings, namely, those that involve an edge that is incident to its special vertex. The second procedure (Lemma 2 removes multiple crossings between edge pairs. Both procedures reduce the overall number of crossings. Hence, they can be exhaustively applied to obtain a redrawing $\Gamma^{\prime}$ of $\Gamma$ that does not contain multiple crossings between edge pairs and where adjacent crossings only involve edges that are not incident to their special vertices (Corollary 4). The procedure (Lemma 4 ) for removing these remaining crossings is quite involved and based on a structural analysis (Lemma 3) of the drawing $\Gamma^{\prime}$.

The first procedure, for getting rid of some of the adjacent crossings, is very simple; the proof is deferred to Appendix A but illustrated in Figure 4.

Lemma 1. Let $\Gamma$ be a non-simple fan-planar drawing. Let $b=(B, R)$ be an edge in $\Gamma$ that is incident to its special vertex $B$. If $b$ has at least one crossing, then one of the edges in the drawing can be redrawn such that the total number of crossings in the drawing decreases. Moreover, the redrawing is fan-planar.

We continue by describing the second procedure, which eliminates crossings between pairs of edges (independent or adjacent) that cross more than once.

Lemma 2. Let $\Gamma$ be a non-simple fan-planar drawing. Let $b=(G, R)$ be an edge in $\Gamma$ whose special vertex $B$ is not incident to $b$. If edge $b$ has multiple crossings with at least one other edge, then an edge that crosses $b$ multiple times, say $g=(B, W)$ (where $W$ could also be incident to b), can be redrawn such that at least one crossing between $b$ and $g$ is eliminated and the total number of crossings in the drawing decreases. Moreover, the redrawing is fan-planar.

Proof. We start by describing a procedure to pick the edge that will be redrawn. We traverse $b$ from $G$ to $R$, until the second crossing of an edge $g=(B, W)$ with $b$ is encountered such that the first crossing of $g$ with $b$ appeared before its second crossing, i.e., the second crossing $y$ with $b$ is closer to $R$ than its first crossing $x$ with $b$, see Figure $5(\mathrm{a})$. If no such edge exists, we exchange the roles of $R$ and $G$ and repeat the procedure. We are guaranteed to find an edge $g$ with the desired properties, since there is an edge crossing $b$ multiple times.

So without loss of generality, assume that the edge $g$ has its second crossing $y$ with $b$ closer to $R$ than its first crossing $x$. We then walk from $y$ towards $G$ along $b$ until we encounter a crossing $z$ between an edge $p$ and $b$. The edge $p$ must also be incident to $B$, the special vertex of $b$.

We can now describe the redrawing procedure. The edge $g$ is redrawn to follow its previous drawing from $W$ to $y$, cross $b$ at $y$, follow the drawing of $b$ 


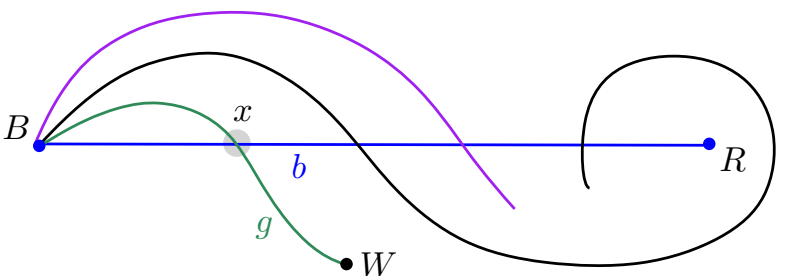

(a)

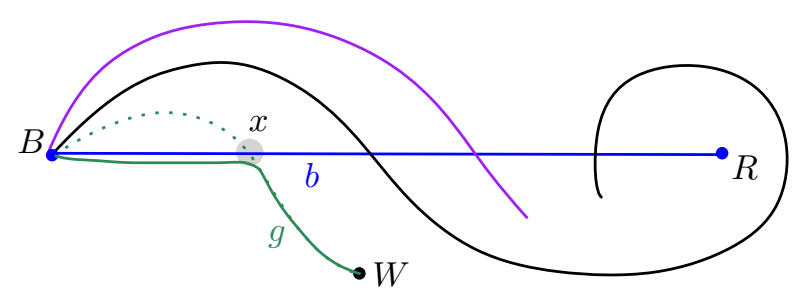

(b)

Fig. 4. Illustration of Lemma 1 If $b$ is incident to its special vertex $B$, then all crossings on $b$ are adjacent crossings. We redraw the edge $g$ whose crossing $x$ with $b$ is closest to $B$ along $b$. Redrawing the part of $g$ between $x$ and $B$ along $b$ cannot introduce any new crossings.

from $y$ to $z$, and, finally, closely follow $p$ from $z$ to $B$; for an illustration see Figure 5 The following statements are proved in Appendix B.

Proposition 1. The crossing $z$ is the first crossing of $p$ with $b$.

Proposition 2. Redrawing g maintains fan-planarity. Moreover, there is an injective mapping that assigns each crossing on the redrawn part of $g$ to a crossing on the replaced part of $g$ that involves the same edges.

The described redrawing of $g$ eliminates the crossing between $g$ and $b$ at $x$. Moreover, by Proposition 1, the redrawn version of $g$ does not contain any new crossings between $b$ and $g$. Combined with Proposition 2 , it follows that the total number of crossings decreases. Moreover, fan-planarity is maintained.

Equipped with Lemmata 1 and 2, we can apply the following normalization to the drawing (the proof can be found in Appendix C):

Corollary 4. Let $\Gamma$ be a non-simple fan-planar drawing. There is a fan-planar redrawing $\Gamma^{\prime}$ of $\Gamma$ such that

- no two edges cross more than once in $\Gamma^{\prime}$;

- no edge is incident to its special vertex; and

- $\Gamma^{\prime}$ does not have more crossings than $\Gamma$. 


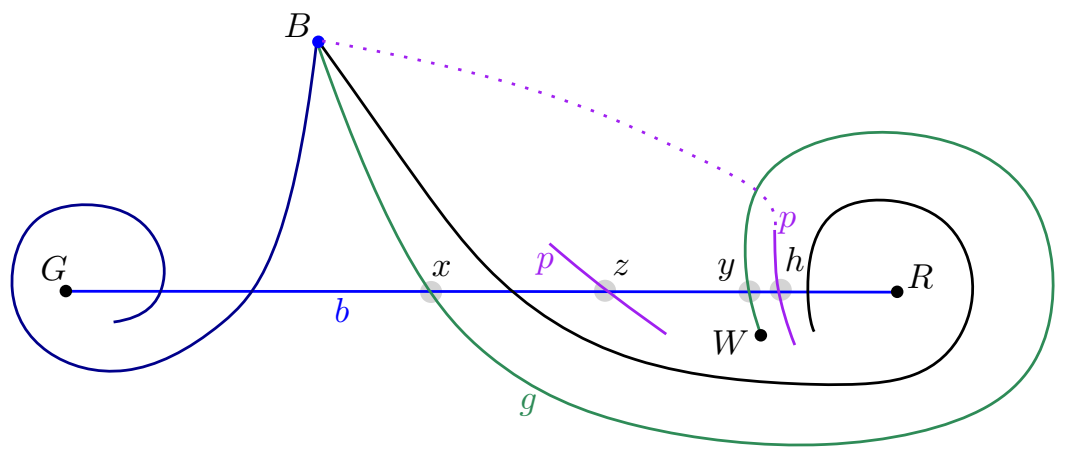

(a)

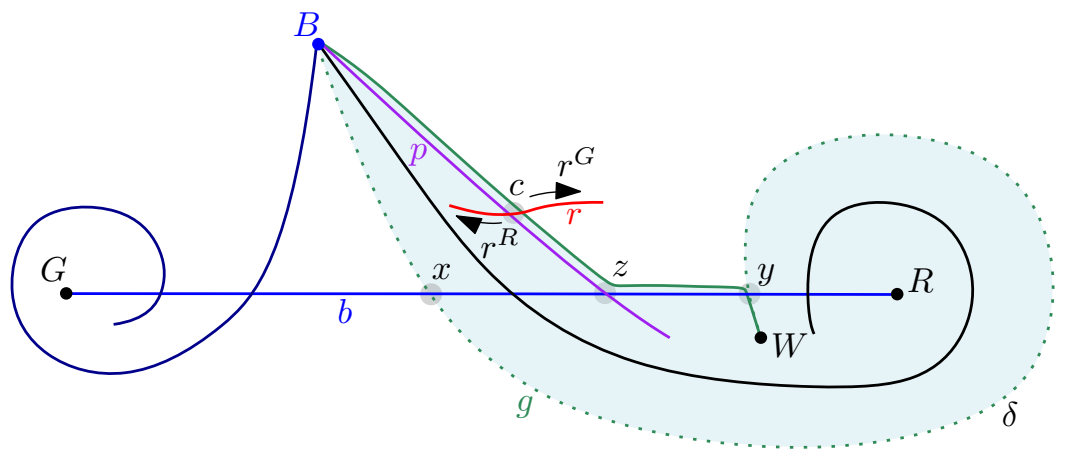

(b) Redrawing of edge $g$.

Fig. 5. Edges $b$ and $g$ cross multiple times and the special vertex $B$ of $b$ is not incident to $b$. The figures do not reflect the case when $W$ is incident to $b$. Any new crossing with the redrawn version of $g$ involves an edge $r$ crossing $p$ between $z$ and $B$, which has to cross the replaced part of $g$ since it is incident to $G$ or $R$.

Adjacent crossings between edges that are not incident to their special vertices may lead to configurations where the previous edge-rerouting strategies would incur additional crossings. In the following lemma, we deal with some unproblematic cases and characterize the remaining, more challenging, configurations in terms of a sequence of conflicting edges.

Lemma 3. Let $\Gamma$ be a non-simple fan-planar drawing in which no two edges cross more than once and such that no edge is incident to its special vertex. Let $b=(G, R)$ and $g=(R, B)$ be (adjacent) edges which cross each other at $x$.

We can redraw $g$ such that the total number of crossings decreases and fanplanarity is maintained; or, alternatively, we can determine a sequence of edges $r_{0}, b_{1}, r_{2}, b_{3}, r_{4}, \ldots, r_{k}$ such that the edges $b, g, r_{0}, b_{1}, r_{2}, b_{3}, r_{4}, \ldots, r_{k}$ are pairwise distinct and the following properties are satisfied (we call the edges $r_{i}$ "red" and the edges bi "black"; for an illustration, see Figure 6, as well as Figure 8, which also depicts $r_{k}$ ): 
$I 1 B$ is the special vertex of the black edges and incident to the red edges.

I2 $R$ is the special vertex of the red edges and incident to the black edges.

I3 For any odd $i$, the first crossing $x_{i+1}$ of $b_{i}$ starting from $R$ is with $r_{i+1}$. For any even $i<k$, the first crossing $x_{i+1}$ of $r_{i}$ starting from $B$ is with $b_{i+1}$.

$I_{4} r_{0}$ crosses $b_{1}$ but no other black edge. $b$ crosses $r_{0}$ and $r_{k}$ but no other red edges.

I5 For the purposes of the final two invariants, we define $q_{-1}=b$. For $0 \leq i<k$, let $\alpha_{i}$ be the closed curve defined by $g$, the arc of $q_{i}$ and the arc of $q_{i-1}$, where $q \in\{r, b\}$, that connect $R, B$ and $x_{i}$. For $0 \leq i<k$, let $\Gamma_{i}$ be the drawing induced by the edges $b, g, r_{0}, b_{1}, r_{2}, \ldots, q_{i}$.

For $0 \leq i<k$, the curve $\alpha_{i}$ is simple and bounds a region $f_{i}$ that contains only $G$, an arc of b that connects $G$ to $x \in \alpha_{i}$ and, possibly, an arc of $r_{0}$ that connects $G$ to $\alpha_{i}$, in its interior.

I6 For $0<i<k, f_{i} \subset f_{i-1}$ and $f_{i-1} \backslash f_{i}$ is an empty triangular face in $\Gamma_{i}$ bounded by the following three arcs:

- the arc of $q_{i}$ between $x_{i}$ and the special vertex of $q_{i-1}$,

- the arc of $q_{i-1}$ between $x_{i}$ and $x_{i-1}$,

- the arc of $q_{i-2}$ between $x_{i-1}$ and the special vertex of $q_{i-1}$ where $q \in\{r, b\}$.

Remark 1 . Note that invariant I5 implies that in $\Gamma_{i}, g$ crosses only $b$ and possibly $r_{0}$. Moreover, the arcs of $q_{i}$ and $q_{i-1}$ connecting $R$ and $B$ via $x_{i}$ are uncrossed in $\Gamma_{i}$.

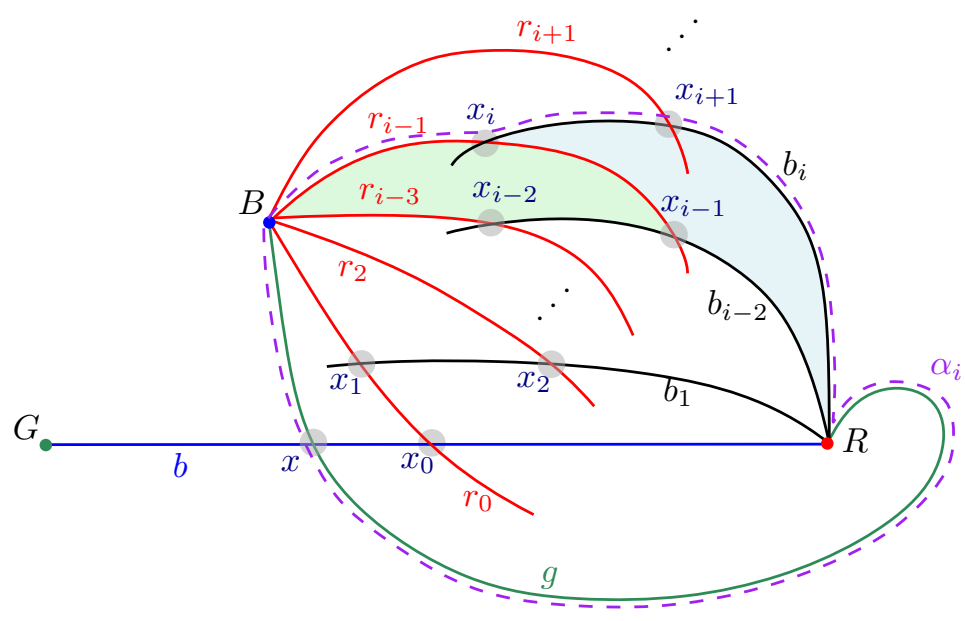

Fig. 6. An example of the sequence of edges described in Lemma 3 . The face $f_{i}$ is the unbounded region delimited by the dashed curve, the face $f_{i-1} \backslash f_{i}$ is depicted in blue and the face $f_{i-2} \backslash f_{i-1}$ is depicted in green.

Proof. It follows from the preconditions that $B$ is the special vertex of $b$ and $G$ is the special vertex of $g$. We will construct the sequence of edges inductively. 
Base case. For the induction base case, we show how to determine $r_{0}$ and $b_{1}$ such that all invariants are satisfied with respect to $r_{0}$. For $b_{1}$, we will only establish the invariants I2, I4, I5 and I6.

We traverse from $R$ along $b$ until we encounter an edge $r_{0}$ that crosses $b$ and denote its crossing by $x_{0}$. If $x_{0}=x$ and, hence, $r_{0}=g$, we can redraw the part of $g$ that leads from $R$ to $x$ along $b$ such that the crossing at $x$ is removed. Moreover, since the redrawn part is crossing-free, the total number of crossings is decreased and fan-planarity is maintained. Hence, if $x_{0}=x$, the statement of the lemma holds.

So assume that $x_{0} \neq x$. It follows that, $r_{0} \neq g$ since $g$ cannot cross $b$ multiple times. Moreover, $r_{0} \neq b$ since edges are realized as simple curves. Since $r_{0}$ intersects $b$, it is incident to $B$.

Now, we traverse $r_{0}$ from $B$ towards $x_{0}$ until we encounter a crossing $x_{1}$ with an edge $b_{1}$. If $x_{1}=x_{0}$ and, hence, $b_{1}=b$, we redraw $g$ along the part of $b$ between $R$ and $x_{0}$ and the part of $r_{0}$ between $x_{0}$ and $B$. The redrawn version of $g$ is crossing-free. Hence, we have eliminated at least one crossing (namely $x$ ) while maintaining fan-planarity and, thus, the statement of the lemma holds if $x_{1}=x_{0}$.

So assume that $x_{1} \neq x_{0}$. It follows that $b_{1}$ is distinct from $b$ since $b$ has no multiple crossings with $r_{0}$. Moreover, $b_{1} \neq r_{0}$ since edges are simple curves. Finally, we show that $b_{1} \neq g$. In fact, we actually claim something stronger and prove it in Appendix D

Proposition 3. The part of $r_{0}$ between $B$ and $x_{0}$ cannot cross $g$.

In particular, Proposition 3 implies $b_{1} \neq g$, as claimed. Thus, we have determined two edges $r_{0}$ and $b_{1}$ such that $b, g, r_{0}, b_{1}$ are pairwise distinct. It remains to show that the desired invariants hold. We have already established that $r_{0}$ is incident to $B$ (since it intersects $b$ ) and, thus, I1 is satisfied for $r_{0}$.

Since $b_{1}$ and $b$ cross $r_{0}$, it follows that $b_{1}$ shares an endpoint with $b$, which is the special vertex of $r_{0}$. Accordingly, we consider two cases. First, assume that the special vertex of $r_{0}$ is $G$, which is illustrated in Figure 7. Consider the closed curve $\alpha_{0}$ described by $g$, the part of $r_{0}$ between $x_{0}$ and $B$ and the part of $b$ between $R$ and $x_{0}$. By Proposition 3 and the fact that there are no multiple crossings, the curve $\alpha_{0}$ is indeed simple. Orient $b$ and $b_{1}$ towards $G$. Since the resulting orientation of the crossings $x_{0}$ and $x_{1}$ has to be consistent, it follows that the part of $b_{1}$ that connects $x_{1}$ with $G$ has to intersect $\alpha_{0}$. More specifically, since there are no multiple crossings, it needs to intersect $g$ in some point $z$. We now redraw $g$ along the part of $b$ that connects $R$ with $x_{0}$ and the part of $r_{0}$ that connects $x_{0}$ with $B$. The redrawn version of $g$ only has crossings along the part between $x_{0}$ and $B$. In particular, it crosses $b_{1}$ at $x_{1}$, but the orientation of this crossing is consistent with the orientation of $z$ in the original drawing of $g$. The same argument applies for all other intersected edges. Consequently, we introduce no additional crossings, eliminate the crossing $x$, and maintain fanplanarity. Hence, the statement of the lemma holds if the special vertex of $r_{0}$ is $G$. It remains to consider the case where the special vertex of $r_{0}$ is $R$ and, 
hence, $b_{1}$ is incident to $R$. It follows that invariant 12 is satisfied for both $r_{0}$ and $b_{1}$.

Invariant I3 for $r_{0}$ is satisfied by construction (and for $b_{1}$ there is nothing to show). Invariant I4 is also satisfied for $r_{0}$ and $b_{1}$ by construction.

The edge $r_{0}$ cannot cross $b$ or $b_{1}$ a second time. If it crosses $g$, then it is incident to $G$, the special vertex of $g$. In any case, this implies invariant I5 for $\Gamma_{0}$.

We observe that $b_{1}$ cannot cross $b$ or $g$ since this would imply that $b_{1}$ is incident to $B$ or $G$ (the special vertex of $b$ and $g$, respectively) and hence $b_{1}$ is parallel to $g$ or $b$, respectively. Moreover, $b_{1}$ cannot cross $r_{0}$ a second time. Hence, the part of $b_{1}$ that leads from $R$ to $x_{1}$ is crossing-free in the drawing $\Gamma_{1}$. Together with invariant I5 for $\Gamma_{0}$, the invariant I5 holds for $\Gamma_{1}$ and invariant I6 holds, which concludes the base case.

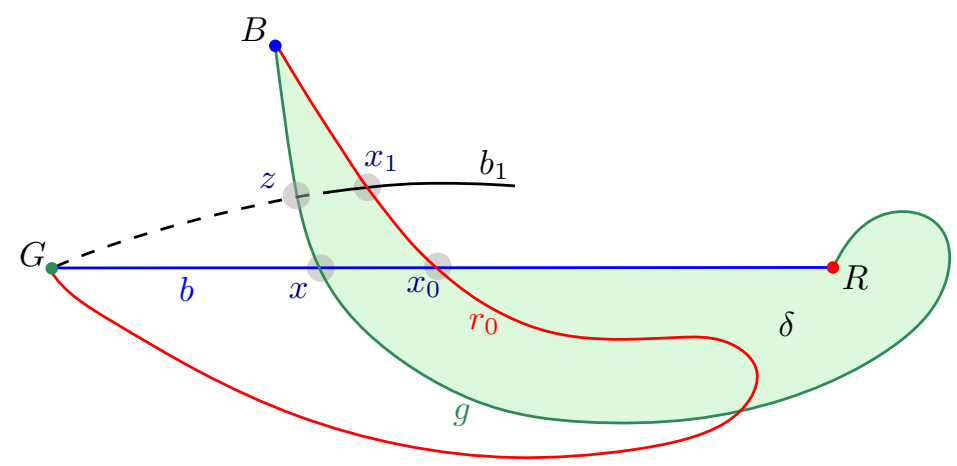

Fig. 7. $r_{0}$ can be incident to $G$. $b_{1}$ is drawn as if $G$ is the special vertex of $r_{0}$.

Inductive Step: Now, assume the first $j+1$ edges, $r_{0}, b_{1}, \ldots, q_{j}$, have been determined and $j<k$. We assume all invariants hold for $r_{0}, \ldots q_{j-1}$. Additionally, we assume that I1. I4. I5 and I6 hold for $q_{j}$ if $j$ is even (and hence $q_{j}=r_{j}$ is red), or I2, I4, I5 and I6 hold for $q_{j}$ if $j$ is odd (and hence $q_{j}=b_{j}$ is black).

We will now determine the edge $q_{j+1}$. If $j$ is even, we need to prove the invariants I2 and I3 for $q_{j}$ and the invariants I2, I4, I5 and I6 for $q_{j+1}$. If $j$ is odd, we need to prove the invariants I1 and I3 for $q_{j}$ and the invariants I1. I4. 15 and 16 (and 12 and I3 if $j+1=k$ ) for $q_{j+1}$.

Case 1: $q_{j}=r_{j}$. Note that in this case, we have nothing to prove for invariant I1.

If the edge $r_{j}$ has no crossings between $B$ and $x_{j}$, then we could redraw $g$ along this part of $r_{j}$ and the arc of $b_{j-1}$ from $x_{j}$ to $R$. The redrawn version of $g$ would then be uncrossed by invariant $\left[3\right.$ for $b_{j-1}$ and the lemma is proved.

Otherwise $r_{j}$ has at least one crossing between $B$ and $x_{j}$. We determine edge $b_{j+1}$ as follows: traverse along $r_{j}$ from $B$ towards $x_{j}$ until we encounter the first edge that crosses $r_{j}$, let this edge be $b_{j+1}$. 
Invariant I3 for $r_{j}$ is satisfied by construction. To prove the remaining invariants, we establish several propositions, the proofs of which can be found in Appendix E. First we prove invariant I2 for $r_{j}$ and $b_{j+1}$.

Proposition 4. Edge $b_{j+1}$ is incident to $R$.

Since $r_{j}$ crosses both edges $b_{j+1}$ and $b_{j-1}$, which are both incident to $R$, the special vertex of $r_{j}$ is $R$, which proves invariant I2.

Next we prove invariant I4. We first need to prove $b_{j+1}$ is distinct from all the previous edges.

Proposition 5. $b_{j+1}$ is distinct from all edges of $\Gamma_{j}$ and does not cross edge $r_{0}$.

Lastly, to prove invariants I5 and I6, we have to prove the following proposition.

Proposition 6. The arc of $b_{j+1}$ between $R$ and $x_{j+1}$ is uncrossed in the drawing $\Gamma_{j+1}$.

So the arc of $b_{j+1}$ between $x_{j+1}$ and $R$ is uncrossed in the drawing $\Gamma_{j+1}$. Further, the arc of $r_{j}$ between $x_{j}$ and $B$ was uncrossed in $\Gamma_{j}$ as noted in Remark 1 . Since $b_{j+1}$ is the only new edge introduced for $\Gamma_{j+1}$, the arcs of $r_{j}$ between $x_{j}$ and $x_{j+1}$ as well as between $x_{j+1}$ and $B$ are uncrossed in $\Gamma_{j+1}$. The latter in conjunction with the above proposition yields that indeed, there is a face $f_{j+1}$ admitting invariant I5. To see this, note that $b_{j+1}$ cannot cross $g$, since it is not incident to $G$ (otherwise it would be parallel to $b$ ). Therefore, no additional edges cross $g$ while extending the subdrawing from $\Gamma_{j}$ to $\Gamma_{j+1}$. Invariant $I 5$ can be combined with the fact that the arc of $b_{j-1}$ from $R$ to $x_{j}$ is uncrossed by invariant $I 3$ to conclude that the triangular region $f_{j} \backslash f_{j+1}$ is indeed empty and invariant $\overline{\mathrm{I}}$ is established.

This concludes the proof of the lemma in the case when $q_{j}=r_{j}$. The second case, $q_{j}=b_{j}$, is similar to the first one and can be found in Appendix F.

Now that we concluded the proof of Lemma 3 , we have all the tools to prove Lemma 4

Lemma 4. Let $\Gamma$ be a non-simple fan-planar drawing with the properties established by Corollary 4. If there is an edge $b=(G, R)$ in $\Gamma$ that crosses an edge $g$ at $x$ and $g$ is incident to $R$, then we can redraw an edge such that the total number of crossings in $\Gamma$ decreases, and the drawing remains fan-planar.

Proof. Let $b=(G, R)$ and $g=(B, R)$ be two adjacent edges which cross at $x$. Their common endpoint is not the special vertex of either of the edges by Corollary 4 . Thus, the special vertices of $b$ and $g$ must be $B$ and $G$, respectively. We apply Lemma3 3 on $b$ and $g$. If $g$ can be redrawn using Lemma 3, this concludes the proof of Lemma 4. Assume that $g$ cannot be redrawn. Then we can determine a sequence of edges $r_{0}, b_{1}, r_{2}, \ldots, r_{k}$ with the properties described in Lemma 3 . We now describe how the edge $b$ can be redrawn to eliminate the crossing $x$ while maintaining fan-planarity and decreasing the overall number of crossings. 
Let the other endpoint of edge $r_{k}$ be $W$. By invariant $14, r_{k}$ has a crossing with edge $b$. First assume this crossing occurs between $x_{k}$ and $W$, i.e., after $r_{k}$ enters the triangular region $f_{k-2} \backslash f_{k-1}$ at $x_{k}$. Since $b$ does not enter this region, $r_{k}$ has to leave it. It cannot cross $b_{k-1}$ again, nor can it cross $r_{k-2}$, because it is not incident to its special vertex $R$ (note that $W \neq R$ since otherwise $r_{k}$ would be parallel to $g$ ). Finally, it cannot cross $b_{k-3}$, because this is the part of $b_{k-3}$ that is uncrossed by invariant $I 3$. Hence, the crossing of $r_{k}$ and $b$ cannot lie between $x_{k}$ and $W$ and must instead lie between $B$ and $x_{k}$ along $r_{k}$. In this case, we claim that edge $b$ can be redrawn. Redraw edge $b$ to follow $g$ from $R$ until $x$, and then follow its previous drawing from $x$ until $G$ while avoiding crossing $g$ at $x$, as illustrated in Figure 8. We now prove that this redrawing does not introduce any new crossings on $b$.

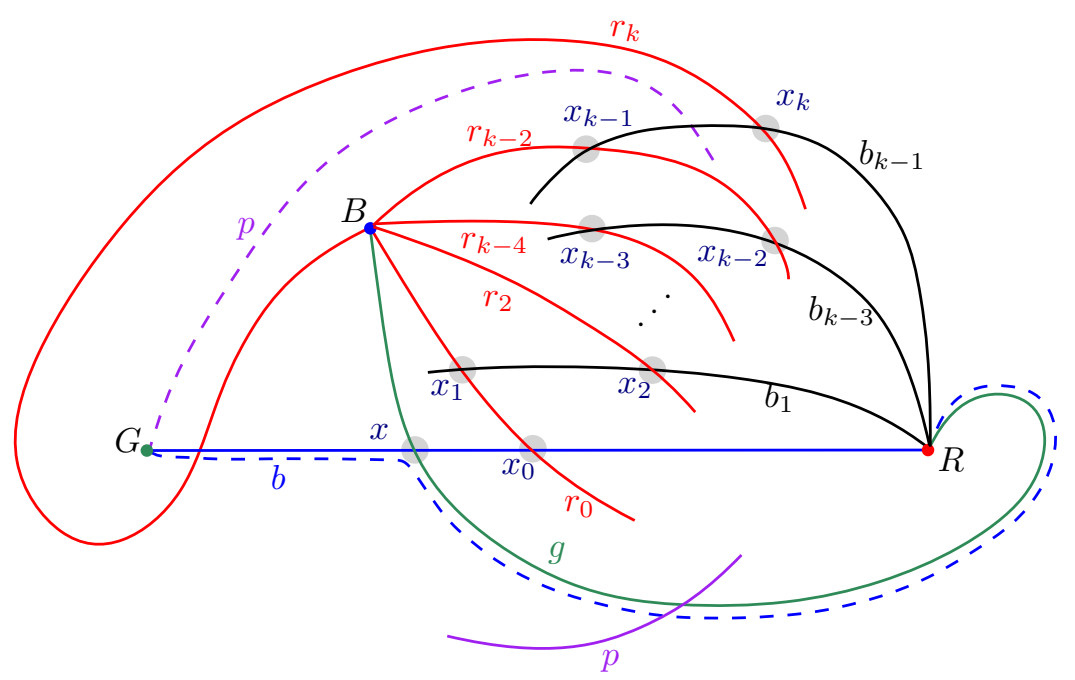

Fig. 8. Redrawing of edge $b$.

Proposition 7. Redrawing $b$ does not introduce any new crossings on $b$.

Proof. Assume a new crossing with an edge $p$ is introduced on $b$ by the redrawing operation. Since the redrawn part of $b$ is parallel to a part of $g$, the edge $p$ crosses $g$ as well. Consequently, edge $p$ is incident to $G$, the special vertex of $g$.

Consider the closed curve $\delta$ formed by the arc of $r_{k}$ between $B$ and $x_{k}$, the arc of $r_{k-2}$ between $B$ and $x_{k-1}$, and the arc of $b_{k-1}$ between $x_{k-1}$ and $x_{k}$. The edge $b$ crosses $r_{k}$ exactly once, does not cross $r_{k-2}$ due to invariant I4, and also does not cross $b_{k-1}$ since the special vertex of $b_{k-1}$ is $B$ due to invariant [1] and $b$ is not incident to $B$. This implies that $b$ crosses $\delta$ exactly once and thus $R$ and $G$ have to lie on distinct sides of $\delta$. This is illustrated in Figure 8, Edge $g$ does not cross any of the edges on the boundary of $\delta$ since $b$ is the only edge crossed 
by $g$ by Remark 1 except possibly for $r_{0}$, and even if $r_{k-2}=r_{0}$ the part of $r_{k-2}$ on $\delta$ is still uncrossed by invariant [3, and therefore $g$ is contained in the same side of $\delta$ as its endpoint $R$.

The edge $p$ crosses $g$ and is incident to $G$, and thus must cross the curve $\delta$ since $g$ and $G$ lie on distinct sides of $\delta$. Edge $p$ cannot be incident to $R$ since then $p$ would be parallel to $b$. Since $R$ is the special vertex of $r_{k}$ and $r_{k-2}$ and $p$ is not incident to $R, p$ cannot cross the edges $r_{k}$ and $r_{k-2}$. Hence, $p$ must cross edge $b_{k-1}$ to cross the curve $\delta$. Then the other endpoint of $p$ must be $B$, the special vertex of $b_{k-1}$. However, the part of $p$ connecting $G$ with $b_{k-1}$ is on the same side as the part of $r_{k-2}$ between $B$ and $b_{k-1}$. Consequently, the part of $p$ connecting $B$ to $b_{k-1}$ and the part of $r_{k-2}$ between $B$ and $b_{k-1}$ lie on distinct sides of $b_{k-1}$, which contradicts the fan-planarity. Overall, we have shown that $p$ cannot cross $\delta$ and, by extension, it cannot cross $g$; a contradiction.

The only redrawn edge is $b$ and no new crossing is introduced on $b$, which ensures that fan-planarity is maintained. Additionally, we eliminate the crossing $x$, which decreases the total number of crossings in the drawing.

We already described in the beginning of Section 3 how Lemmata 14 can be combined to obtain a proof of Theorem 1] we formally summarize the proof in Appendix G.

\section{References}

1. Ackerman, E., Tardos, G.: On the maximum number of edges in quasi-planar graphs. J. Comb. Theory, Ser. A 114(3), 563-571 (2007). https://doi.org/10.1016/j.jcta.2006.08.002

2. Agarwal, P.K., Aronov, B., Pach, J., Pollack, R., Sharir, M.: Quasiplanar graphs have a linear number of edges. Comb. 17(1), 1-9 (1997). https://doi.org/10.1007/BF01196127

3. Angelini, P., Bekos, M.A., Kaufmann, M., Kindermann, P., Schneck, T.: 1fan-bundle-planar drawings of graphs. Theor. Comput. Sci. 723, 23-50 (2018). https://doi.org/10.1016/j.tcs.2018.03.005

4. Bae, S.W., Baffier, J., Chun, J., Eades, P., Eickmeyer, K., Grilli, L., Hong, S., Korman, M., Montecchiani, F., Rutter, I., Tóth, C.D.: Gap-planar graphs. Theor. Comput. Sci. 745, 36-52 (2018). https://doi.org/10.1016/j.tcs.2018.05.029

5. Bekos, M.A., Cornelsen, S., Grilli, L., Hong, S., Kaufmann, M.: On the recognition of fan-planar and maximal outer-fan-planar graphs. Algorithmica 79(2), 401-427 (2017). https://doi.org/10.1007/s00453-016-0200-5

6. Bekos, M.A., Grilli, L.: Fan-planar graphs. In: Hong, S., Tokuyama, T. (eds.) Beyond Planar Graphs, Communications of NII Shonan Meetings, pp. 131-148. Springer (2020). https://doi.org/10.1007/978-981-15-6533-5_8

7. Bekos, M.A., Kaufmann, M., Raftopoulou, C.N.: On optimal 2- and 3-planar graphs. In: Aronov, B., Katz, M.J. (eds.) 33rd International Symposium on Computational Geometry, SoCG 2017, July 4-7, 2017, Brisbane, Australia. LIPIcs, vol. 77, pp. 16:1-16:16. Schloss Dagstuhl - Leibniz-Zentrum für Informatik (2017). https://doi.org/10.4230/LIPIcs.SoCG.2017.16 
8. Binucci, C., Chimani, M., Didimo, W., Gronemann, M., Klein, K., Kratochvíl, J., Montecchiani, F., Tollis, I.G.: Algorithms and characterizations for 2-layer fanplanarity: From caterpillar to stegosaurus. J. Graph Algorithms Appl. 21(1), 81102 (2017). https://doi.org/10.7155/jgaa.00398

9. Binucci, C., Giacomo, E.D., Didimo, W., Montecchiani, F., Patrignani, M., Symvonis, A., Tollis, I.G.: Fan-planarity: Properties and complexity. Theor. Comput. Sci. 589, 76-86 (2015). https://doi.org/10.1016/j.tcs.2015.04.020

10. Brandenburg, F.J.: On fan-crossing graphs. Theor. Comput. Sci. 841, 39-49 (2020). https://doi.org/10.1016/j.tcs.2020.07.002

11. Cheong, O., Har-Peled, S., Kim, H., Kim, H.: On the number of edges of fan-crossing free graphs. Algorithmica 73(4), 673-695 (2015). https://doi.org/10.1007/s00453-014-9935-z

12. Didimo, W., Eades, P., Liotta, G.: Drawing graphs with right angle crossings. Theor. Comput. Sci. 412(39), 5156-5166 (2011). https://doi.org/10.1016/j.tcs.2011.05.025

13. Didimo, W., Liotta, G., Montecchiani, F.: A survey on graph drawing beyond planarity. ACM Comput. Surv. 52(1), 4:1-4:37 (2019). https://doi.org/10.1145/3301281

14. Holten, D.: Hierarchical edge bundles: Visualization of adjacency relations in hierarchical data. IEEE Trans. Vis. Comput. Graph. 12(5), 741-748 (2006). https://doi.org/10.1109/TVCG.2006.147

15. Holten, D., van Wijk, J.J.: Force-directed edge bundling for graph visualization. Comput. Graph. Forum 28(3), 983-990 (2009). https://doi.org/10.1111/j.14678659.2009.01450.x

16. Hong, S., Tokuyama, T. (eds.): Beyond Planar Graphs, Communications of NII Shonan Meetings. Springer (2020). https://doi.org/10.1007/978-981-15-6533-5

17. Kaufmann, M., Ueckerdt, T.: The density of fan-planar graphs. CoRR abs/1403.6184v1 (2014), http://arxiv.org/abs/1403.6184v1

18. Kaufmann, M., Ueckerdt, T.: The density of fan-planar graphs. CoRR abs/1403.6184v2 (2014), http://arxiv.org/abs/1403.6184v2

19. Pach, J., Tóth, G.: Graphs drawn with few crossings per edge. Comb. 17(3), 427439 (1997). https://doi.org/10.1007/BF01215922

20. Telea, A.C., Ersoy, O.: Image-based edge bundles: Simplified visualization of large graphs. Comput. Graph. Forum 29(3), 843-852 (2010). https://doi.org/10.1111/j.1467-8659.2009.01680.x 


\section{A Proof of Lemma 1}

Proof. Every edge that crosses $b$ is incident to $B$. Traverse along the edge $b$ from $B$ to $R$, until an edge $g=(B, W)$ that crosses $b$ is encountered. Let this crossing be $x$. We redraw the edge $g$ to follow the drawing of $b$ from $B$ until $x$ and then follow its previous drawing from $x$ to $W$ without crossing $b$ at $x$. The rerouting is illustrated in Figure 9 .

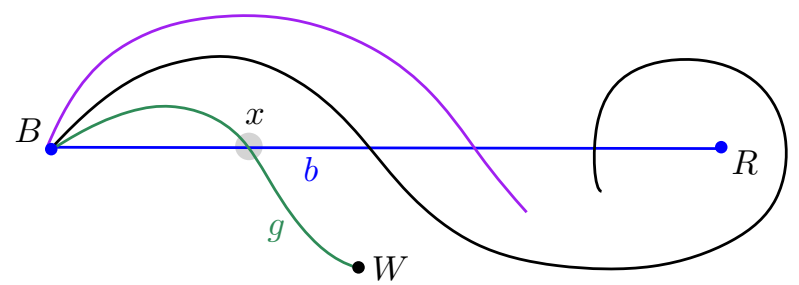

(a) Edge $g$ is adjacent to $b$ and crosses $b$.

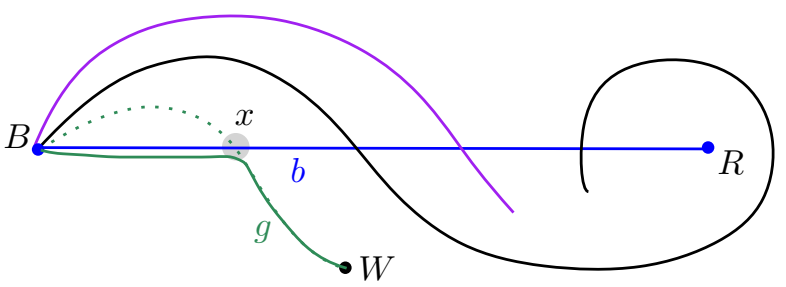

(b) Redrawing of edge $g$.

Fig. 9. Illustration of Lemma 1 If $b$ is incident to its special vertex $B$, then all crossings on $b$ are adjacent crossings. We redraw the edge $g$ whose crossing $x$ with $b$ is closest to $B$ along $b$. Redrawing the part of $g$ between $x$ and $B$ along $b$ cannot introduce any new crossings.

The part of edge $b$ between $B$ and $x$ has no crossings by definition of $x$. Hence, the rerouting introduces no new crossings. In particular, no new crossings are introduced on $g$ and, hence, fan-planarity is maintained. Finally, since the crossing between $b$ and $g$ at $x$ is eliminated, the total number of crossings decreases.

\section{B Proofs of the propositions in Lemma 2}

Proof (of Proposition 11). If $p=g$, then we claim that $x=z$, i.e., $z$ is also the first crossing of $p(=g)$ with $b$. Assume otherwise that $z$ is the $i^{\text {th }}$ crossing of $p(=g)$ with $b$, where $i>2$. Consider the closed curve $\delta$ formed by the arcs of $b$ and $g$ between $x$ and $y$. The arc of $p(=g)$ between $y$ and $W$ must cross the curve $\delta$ at $z$. However, this implies that this arc of $p$ crosses itself or it crosses $b$ such that orienting $p$ towards $B$ and $b$ arbitrarily does not result in a consistent 
orientation of crossings. In both cases, we obtain a contradiction and, hence, $z$ is indeed the first crossing of $p(=g)$ with $b$ if $p=g$.

Now assume $p \neq g$ and assume that the crossing at $z$ is the $i^{\text {th }}$ crossing of $p$ with $b$, where $i \geq 2$. Observe that in a fan-planar drawing, if an edge $e$ has three crossings $x_{i}, x_{j}, x_{k}$ with an edge $f$ such that $x_{\ell}$, where $\ell \in\{i, j, k\}$, is the $\ell^{t h}$ crossing of $e$ with $f$ and $i<j<k$, then $x_{i}, x_{j}, x_{k}$ appear in this order along $f$. Consequently, the first crossing of $p$ with $b$ has to be located between $z$ and $R$. Otherwise, when traversing $b$ from $G$ to $R$, the first crossing of $p$ with $b$, the second crossing of $p$ with $b$, and the second crossing of $g$ with $b$ would be encountered in this order, which contradicts our choice of $g$. More specifically, the first crossing of $p$ with $b$, say $h$, must be between $y$ and $R$ since the arc of $b$ between $z$ and $y$ has no crossings by construction; the situation is illustrated in Figure $5(\mathrm{a})$. Consider the closed curve $\delta$ again. Since $p$ is incident to $B$ and $h$ is the first crossing of $p$ with $b$, the arc of $p$ starting from $B$ must cross $g$ between $x$ and $y$ to enter the region enclosed by $\delta$ before $p$ crosses $b$ at $h$. After $p$ crosses $b$ at $h$, it has to cross $\delta$ again in order to cross $z$ such that the crossing orientation of $z$ and $h$ is consistent. However, this second crossing with $\delta$ implies that the crossings of $p$ with $g$ or the crossings of $p$ with $b$ are not consistently oriented; a contradiction.

Hence, in any case, $z$ is the first crossing of $p$ with $b$.

Proof (of Proposition 2). To show that fan-planarity is maintained, we have to prove that the crossings introduced along the redrawn edge $g$ satisfy the conditions of fan-planarity. Observe that new crossings introduced along $g$ must be between $B$ and $z$ and the involved edges have to cross edge $p$ as well. Let an edge $r$ cross $p$ (and the redrawn version of $g$ ) at a point $c$ between $z$ and $B$. If $p=g$, then fan-planarity is maintained since then the crossings of the redrawing of $g$ are a subset of the crossings on its original drawing.

Assume $p \neq g$. Let $\phi$ be the closed curve formed by the old drawing of $g$ between $B$ and $y$, the arc of $b$ between $y$ and $z$, and the arc of $p$ between $z$ and $B$. The edge $r$ must cross $\phi$ by definition since $c$ lies on $\phi$. Since the second crossing of $g$ with $b$ is closer to $R$ than the first crossing and due to fan-planarity, $R$ and $G$ have to lie on distinct sides of $\phi$. At $c$, we split $r$ into two parts. We use $r^{R}$ to denote the part that enters the side of $\phi$ that contains $R$ - the other part of $r$ is denoted by $r^{G}$.

The special vertex of $p$, say $P$, must be incident to edge $b$ since $b$ crosses $p$, and the edge $r$ must be incident to $P$ since $r$ also crosses $p$. We distinguish two cases, namely $P=G$ and $P=R$. We show that in both cases, $r$ crosses the part between $B$ and $y$ of the original drawing of $g$.

First, assume $P=G$. Since $P(=G)$ must be on a common side of $p$ for $r$ and $b$, the part of $r$ that is incident to $P(=G)$ has to be $r^{R}$. This implies that $r^{R}$ has to cross the curve $\phi$ by definition of $r^{R}$. Let $s$ be the crossing of $r_{R}$ with curve $\phi$ that is closest to $c$ along $r_{R}$. The crossing $s$ cannot lie on the arc of $p$ on $\phi$, since otherwise $r$ and $p$ would cross as in the configuration SF2 which is forbidden. Further, $s$ cannot lie on the arc of $b$ on $\phi$ since this part of $b$ is uncrossed by the definition of $z$. Hence, $s$ must lie on the old drawing of $g$ between $B$ and $y$, i.e., 
along the part of $\phi$ formed by the old drawing of $g$. This implies that $r$ crosses the part between $B$ and $y$ of the original drawing of $g$.

It remains to consider the case that $P=R$. Since $P(=R)$ is on a common side of $p$ for $r$ and $b$, the part of $r$ that is incident to $P(=R)$ has to be $r^{G}$. The arguments why $r$ crosses the part between $B$ and $y$ of the original drawing of $g$ are analogous to those used in the case $P=G$.

We have shown that $r$ crosses the part between $B$ and $y$ of the original drawing of $g$. The corresponding crossing is eliminated when redrawing $g$, and a crossing between $r$ and $g$ is introduced after the redrawing. Hence, even though the redrawn version of $g$ crosses $r$ between $z$ and $B$, the number of crossings does not increase. Moreover, the orientation of the crossings between $r$ and $g$ is consistent with the orientation of the crossings in the redrawn version, i.e., fan-planarity is maintained.

\section{Proof of Corollary 4}

Proof (of Corollary 4). The redrawing procedures guaranteed by Lemma 2 and 1 decrease the number of crossings. Hence, they can be exhaustively applied to $\Gamma$ to obtain a drawing $\Gamma^{\prime}$ with no more crossings than $\Gamma$ such that $\Gamma^{\prime}$ does not satisfy the precondition of Lemma 1 1 or 2 In particular, if an edge is incident to its special vertex, all edges crossing it must be adjacent to it. If there is such an edge, Lemma 1 is applicable. If there is no such edge, we may choose a new special vertex for that edge, which is not incident to it. Hence, $\Gamma^{\prime}$ has the desired properties.

\section{Proof of the proposition in the base case of Lemma 3}

Proof (of Proposition 3). Assume otherwise and consider the closed curve $\gamma$ formed by the parts of $g$ and $b$ that lead from $R$ to $x$. Both $G$ and $B$ are on the same side of $\gamma$ since there are no multiple crossings. Since $r_{0}$ intersects $g$, it follows that $r_{0}$ is incident to the special vertex $G$ of $g$. Let the arc of $r_{0}$ between $B$ and $x_{0}$ be denoted by $r_{0}^{B}$ and the arc between $G$ and $x_{0}$ be denoted by $r_{0}^{G}$. Since $B$ must lie on the same side of $b$ with respect to the two crossings $x$ and $x_{0}$, the $\operatorname{arc} r_{0}^{G}$ must be the arc on the side of $\gamma$ which does not contain $B$. However, for $r_{0}^{G}$ to be incident to $G, r_{0}^{G}$ must cross $\gamma$, thereby crossing $b$ or $g$ a second time (recall that $r_{0}^{B}$ intersects $g$ by assumption), arriving at a contradiction.

\section{E Proof of the proposition in the inductive step of Lemma 3: Case 1}

Proof (of Proposition 4). Assume $b_{j+1}$ is not incident to $R$. Since $r_{j}$ crosses $b_{j-1}$ and $b_{j+1}$, they must have a common endpoint, which is not $R$ by assumption. Let the other endpoint of $b_{j-1}$ be $Z$, which implies $b_{j+1}$ is also incident to $Z$. Let the arc of $b_{j+1}$ which exits the face $f_{j}$ and enters region $f_{j-1} \backslash f_{j}$ (which 


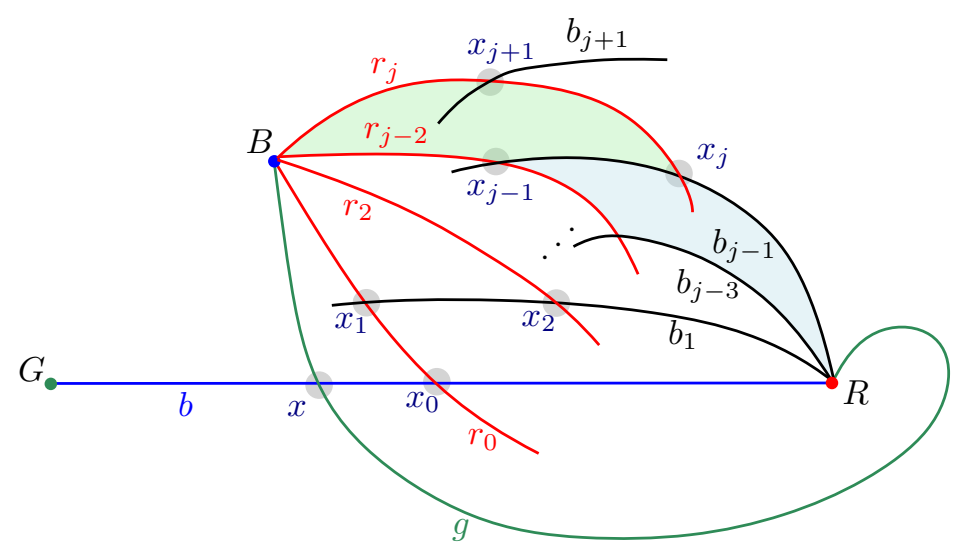

Fig. 10. Determining $b_{j+1}$.

is shaded in green in Figure $10 p$ be denoted by $b_{j+1}^{i}$. To maintain fan-planarity, the vertex $Z$ must be on the same side of $r_{j}$ along both edges $b_{j-1}$ and $b_{j+1}$, which implies that the arc $b_{j+1}^{i}$ must end at $Z$. Endvertex $Z$ is not in the region $f_{j-1} \backslash f_{j}$ by invariant 16 , hence the arc $b_{j+1}^{i}$ must exit the region $f_{j-1} \backslash f_{j}$. To exit the region $f_{j-1} \backslash f_{j}$, arc $b_{j+1}^{i}$ must cross $r_{j}, b_{j-1}$ or $r_{j-2}$. Arc $b_{j+1}^{i}$ cannot cross $r_{j}$ again, and also cannot cross $r_{j-2}$ since the arc of $r_{j-2}$ in this region has no crossings by invariant I3. Hence, $b_{j+1}^{i}$ must cross $b_{j-1}$ to exit the region $f_{j-1} \backslash f_{j}$, and must enter the region $f_{j-2} \backslash f_{j-1}$ (which is shaded in blue in Figure 10. Again, endpoint $Z$ is not in the region $f_{j-2} \backslash f_{j-1}$ by invariant I6. and hence the arc $b_{j+1}^{i}$ must exit the region $f_{j-2} \backslash f_{j-1}$ by crossing $b_{j-1}, b_{j-3}$ or $r_{j-2}$. However, $b_{j+1}^{i}$ cannot cross $b_{j-1}$ again, cannot cross $b_{j-3}$ since the arc of $b_{j-3}{ }^{8}$ in this region is uncrossed by invariant I3 and also cannot cross $r_{j-2}$ since $b_{j+1}$ must be incident to $R$ to cross $r_{j-2}$ since $R$ is the special vertex of $r_{j-2}$. Hence, $b_{j+1}^{i}$ cannot exit the region $f_{j-2} \backslash f_{j-1}$ and thus cannot be incident to $Z$. We arrive at a contradiction, which implies $b_{j+1}$ is incident to $R$.

Proof (of Proposition 5). Edge $b_{j+1}$ is incident to $R$, let the other endpoint be $S$. Let the arc of $b_{j+1}$ which exits the face $f_{j}$ at $x_{j+1}$ and enters region $f_{j-1} \backslash f_{j}$ be denoted by $b_{j+1}^{i}$. To exit the region $f_{j-1} \backslash f_{j}$, arc $b_{j+1}^{i}$ would have to cross $r_{j}$, $b_{j-1}$ or $r_{j-2}$. Arc $b_{j+1}^{i}$ cannot cross $r_{j}$ again, and also cannot cross $r_{j-2}$ since the arc of $r_{j-2}$ in this region is uncrossed by invariant I3 For arc $b_{j+1}^{i}$ to cross $b_{j-1}$, edge $b_{j+1}$ must be incident to $B$, the special vertex of $b_{j-1}$. However, $b_{j+1}$ would be parallel to $g$ in that case and the graph has no parallel edges, so this would only be possible if $g=b_{j+1}$, but $g$ does not cross $b_{j-1}$ either, which implies arc $b_{j+1}^{i}$ cannot cross $b_{j-1}$. Thus, the arc $b_{j+1}^{i}$ cannot exit the region $f_{j-1} \backslash f_{j}$. This shows that the region $f_{j-1} \backslash f_{j}$ contains an endpoint of $b_{j+1}$. Since this region was empty in $\Gamma_{j}$ by invariant I6, $b_{j+1}$ is distinct from all the previous edges.

${ }^{8}$ If $j=2$ this denotes $b_{-1}=b$ again 


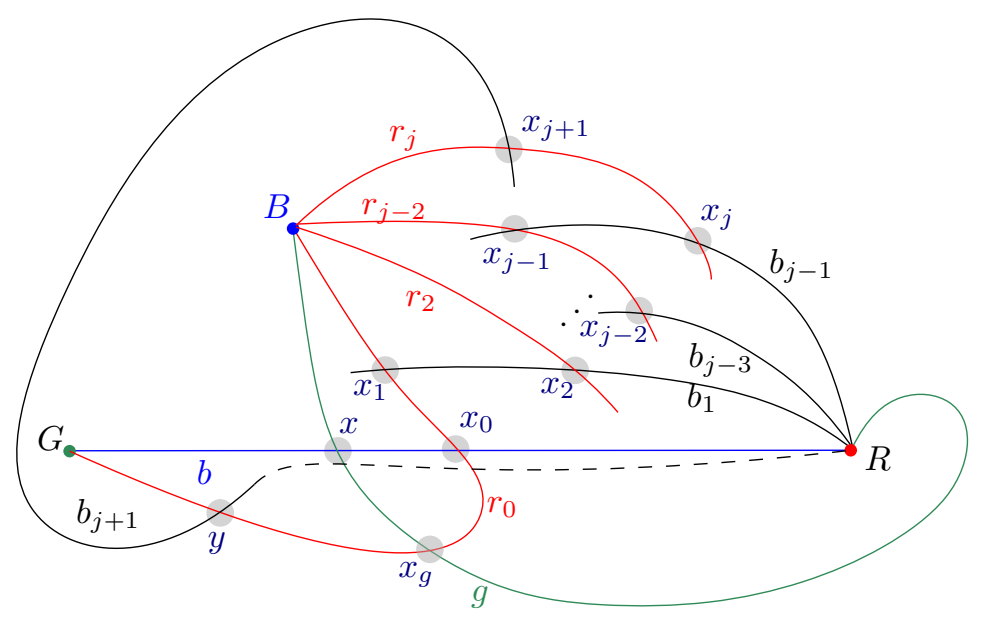

Fig. 11. Illustrates the case that $b_{j+1}$ crosses $r_{0}$ directly after $x_{j+1}$.

Assume edge $b_{j+1}$ crosses edge $r_{0}$. If the arc $b_{j+1}^{i}$ crosses $r_{0}$, the crossing must be in region $f_{j-1} \backslash f_{j}$. This region was empty in $\Gamma_{j}$ though, so $r_{0}$ does not enter it. This also shows $b_{j+1}$ is not incident to $B$ or $G$.

Therefore $r_{0}$ has to cross $b_{j+1}$ along the arc of $b_{j+1}$ between $R$ and $x_{j+1}$ at a crossing $y$. We know $b_{j+1}$ is not incident to $G$ nor $B$, so it doesn't cross $g$ nor $b_{j-1}$. Therefore this arc lies entirely in $f_{j}$, since none of the $\operatorname{arcs} b_{j-1}, r_{j}$ and $g$ can be crossed by it. $r_{0}$ leaves $f_{1} \supset f_{j}$ when crossing $b_{1}$ at $x_{1} . r_{0}$ has to cross $\alpha_{1}$ again in order to re-enter $f_{1}$. To cross $\alpha_{1}$ again, $r_{0}$ has to cross $g$, since it cannot cross itself nor $b_{1}$ again. However, if $r_{0}$ crosses $g$ at a crossing $x_{g}$, the other end point of $r_{0}$ must be $G$. The edge $r_{0}$ thus will enter $f_{1}$ again after crossing $g$ at $x_{g}$, crosses $b_{j+1}$ at $y$ and then ends to $G$. Consider the arc of $b_{j+1}$ that starts at $y$ and ends at $R$. After crossing $r_{0}$ at $y$, this arc enters the triangle $x, x_{g}, G$ bounded by $b, g$ and $r_{0}$, see Figure 11 for an illustration. However, it is impossible for this arc to exit the triangle since none of the edges bounding the triangle can be crossed (again) by $b_{j+1}$ as shown above, and thus the arc of $b_{j+1}$ cannot be incident to $R$, a contradiction.

Proof (of Proposition 6). Towards a contradiction assume that the arc of $b_{j+1}$ between $R$ and $x_{j+1}$ is crossed in $\Gamma_{j+1}$. Then it must be crossed by $b, g$, or $q_{i}$, where $0 \leq i<k, q \in\{r, b\}$.

Let $y$ be the crossing along the arc of $b_{j+1}$ between $x_{j+1}$ and $R$ that is closest to $x_{j+1} \cdot b_{j+1}$ is neither incident to $G$ nor $B$, since it otherwise would be parallel to $b$ or $g$ respectively. Therefore $y$ cannot lie on any edge with these special vertices. This excludes the black edges, $b$ and $g$. By Proposition $5, b_{j+1}$ also does not cross $r_{0}$.

Further $y$ cannot lie on the arc of $r_{j}$ between $B$ and $x_{j+1}$ since this arc is uncrossed by invariant I3. Thus, $y$ cannot lie on the curve $\alpha_{j}$, and must lie in the interior of $f_{j}$. 
Since $b_{j+1}$ crosses $r_{j}$ at $x_{j+1}$ already, $y$ cannot lie on $r_{j}$.

Hence, $y$ must lie on a red edge $r_{i}$ where $1 \leq i<j$. Let $\delta$ be the closed curve formed by the arc of $b$ between $B$ and $x_{0}$, the arc of $r_{0}$ between $R$ and $x_{0}$, the arc of $r_{j}$ between $B$ and $x_{j}$ and the arc of $b_{j-1}$ between $R$ and $x_{j}$. Invariant I6 applied to $i, i+1, i+2$ shows that the entirety of edge $r_{i}$ is outside $f_{i+2} \supset f_{j}$. Invariant I6 applied to $i, i-1, i-2$ shows that it is completely contained in $f_{i-2} \subset f_{0}$. Now let $\delta=\alpha_{0} \triangle \alpha_{j}$, then edge $r_{i}$ must cross $\delta$ since $y$ lies in $f_{j} \subset f_{0}$, on the other side of the curve. To cross $\delta, r_{i}$ must cross one of the arcs of the curve. $r_{i}$ cannot cross $b$ or $b_{j-1}$ since these arcs are uncrossed by invariant I3, and cannot cross $r_{0}$ or $r_{j}$ since it is not incident to $R$, since invariant I2 would imply it is an edge parallel to $g$. Thus, $y$ does not lie on a red edge.

This proves the proposition that the arc of $b_{j+1}$ between $x_{j+1}$ and $R$ is uncrossed in the drawing $\Gamma_{j+1}$.

\section{F Proof of the inductive step of Lemma 3: Case 2}

Case 2: $q_{j}=b_{j}$. Note that in this case, there is nothing to prove for invariant 12 ,

If the edge $b_{j}$ has no crossings between $R$ and $x_{j}$, then we could redraw $g$ along this part of $b_{j}$ and along the arc of $r_{j-1}$ from $x_{j}$ to $B . g$ would then be uncrossed by invariant I3 and the lemma is proved.

We determine the edge $r_{j+1}$ as follows: traverse along $b_{j}$ from $R$ towards $x_{j}$ until we encounter the first edge which crosses $b_{j}$. Let this edge be $r_{j+1}$. We prove that $r_{j+1}$ satisfies the invariants.

Invariant I3 is true by definition. We now prove invariant I1 for $b_{j}$ and $r_{j+1}$.

Proposition 8. Edge $r_{j+1}$ is incident to $B$.

Proof. Assume $r_{j+1}$ is not incident to $B$. Since $b_{j}$ crosses $r_{j-1}$ and $r_{j+1}$, both the edges $r_{j-1}$ and $r_{j+1}$ must have a common endpoint, which is not $B$ by assumption. Let the other endpoint of $r_{j-1}$ be $Z$, which implies $r_{j+1}$ is also incident to $z_{j-1}$. Let the arc of $r_{j+1}$ which exits the face $f_{j}$ and enters region $f_{j-1} \backslash f_{j}$ (which is shaded in blue in Figure 12 be denoted by $r_{j+1}^{i}$. To maintain fan-planarity, the vertex $Z$ must be on the same side of $b_{j}$ along both edges $r_{j-1}$ and $r_{j+1}$, which implies that the arc $r_{j+1}^{i}$ must end at $Z$. Endvertex $Z$ is not in the region $f_{j-1} \backslash f_{j}$ by invariant I6, hence the arc $r_{j+1}^{i}$ must exit the region $f_{j-1} \backslash f_{j}$. To exit the region $f_{j-1} \backslash f_{j}$, arc $r_{j+1}^{i}$ must cross $b_{j}, r_{j-1}$ or $b_{j-2}$. Arc $r_{j+1}^{i}$ cannot cross $b_{j}$ again, and also cannot cross $b_{j-2}$ since the arc of $b_{j-2}$ in this region is uncrossed by invariant $\mathrm{I} 3$. Hence, $r_{j+1}^{i}$ must cross $r_{j-1}$ to exit the region $f_{j-1} \backslash f_{j}$. If $j>1$, it enters the region $f_{j-2} \backslash f_{j-1}$ (which is shaded in green in Figure 12. Again, endpoint $Z$ is not in the region $f_{j-2} \backslash f_{j-1}$ by invariant I6. and hence the arc $r_{j+1}^{i}$ must exit the region $f_{j-2} \backslash f_{j-1}$ by crossing $r_{j-1}, r_{j-3}$ or $b_{j-2}$. However, $r_{j+1}^{i}$ cannot cross $r_{j-1}$ again, cannot cross $r_{j-3}$ since the arc of $r_{j-3}$ in this region is uncrossed by invariant I3, and also cannot cross $b_{j-2}$ since $r_{j+1}$ must be incident to $B$ to cross $b_{j-2}$ since $B$ is the special vertex of $b_{j-2}$. Hence, $r_{j+1}^{i}$ cannot exit the region $f_{j-2} \backslash f_{j-1}$ and thus cannot be incident to $Z$. 
In the special case $j=1$, if $r_{2}$ crosses $b_{1}$ and then $r_{0}$, then the two edges $r_{0}$ and $b_{1}$ must have a vertex in common and since neither edge is $g$, it cannot be $R$ or $B$. However, orienting $r_{2}$ from $B$ to $Z, R$ is on the left at $x_{2}$ and $B$ is on the right. Therefore the other endpoint of $r_{0}$ and $b_{1}, Z$, will lie on different sides of $r_{2}$ with respect to $r_{0}$ and $b_{1}$, and hence cannot be a special vertex of $r_{2}$. In both cases, we arrive at a contradiction, so $r_{j+1}$ is incident to $B$.

Since $b_{j}$ crosses both edges $r_{j+1}$ and $r_{j-1}$, which are both incident to $B$, the special vertex of $b_{j}$ is $B$, which proves invariant I1

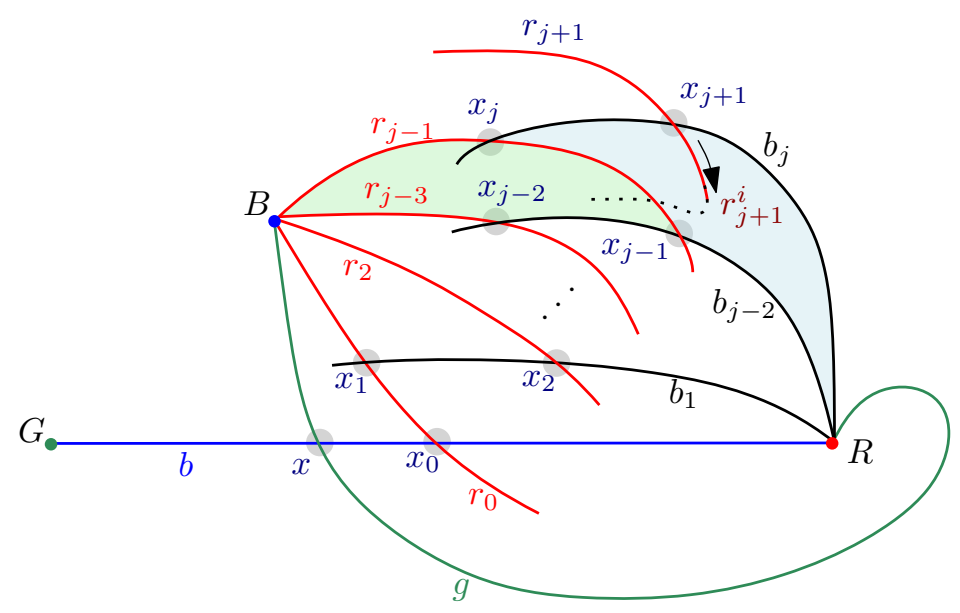

Fig. 12. Determining edge $r_{j+1}$.

Next, we prove that $r_{j+1}$ is distinct from the previous edges.

Proposition 9. $r_{j+1}$ is distinct from the edges of $\Gamma_{j}$.

Proof. Similarly to the last proof, let the arc of $r_{j+1}$ which exits the face $f_{j}$ and enters region $f_{j-1} \backslash f_{j}$ be denoted by $r_{j+1}^{i}$. We claim that this arc cannot leave this region anymore. To do so, it would either have to cross $b_{j}$ again, or cross $b_{j-2}$ in the part uncrossed by invariant I3, or cross $r_{j-1}$. In that case, it is incident to $R$ though, and would therefore be $g$, since there are no parallel edges. It is not $g$ though, because $b_{j}$ does not cross $g$. Therefore, the other endpoint of $r_{j+1}$ lies inside $f_{j-1} \backslash f_{j}$, an empty region in $\Gamma_{j}$, establishing the proposition.

Now, to prove invariants I4] I5, and I6 we prove that $r_{j+1}$ is uncrossed between $x_{j+1}$ and $B$ in the drawing $\Gamma_{j+1}$, unless $j+1=k$.

Proposition 10. If $j+1=k$, then all invariants hold and the arc of $r_{j+1}$ between $x_{j+1}$ and $B$ crosses $b$. Otherwise, this arc is uncrossed in the drawing $\Gamma_{j+1}$. 
Proof. Towards a contradiction assume that the arc of $r_{j+1}$ between $x_{j+1}$ and $B$ is crossed in $\Gamma_{j+1}$. Then it must be crossed by $b, g$ or $q_{i}$, where $i \in\{0, \ldots, k\}$, $q \in\{r, b\}$.

Let $y$ be the crossing along the arc of $r_{j+1}$ between $x_{j+1}$ and $B$ that is closest to $x_{j+1}$. If $y$ lies on the edge $b$, then invariant I4 is satisfied, $i=k$, and the sequence is maximal. Invariants $[5$ and I6 are void in this case, so they hold trivially. Invariants I1 and I3 have already been proved. Finally, invariant $I 2$ is true for $r_{j+1}$, because it crosses $b_{j}$ and $b$, both of which are incident to $R$, so $R$ has to be its special vertex.

Assume $y$ lies on the closed curve $\alpha_{j}$. Recall that the curve $\alpha_{j}$ is formed by the edge $g$, the arc of $b_{j}$ between $R$ and $x_{j}$ and the arc of $r_{j-1}$ between $B$ and $x_{j}$. The crossing $y$ must lie on $g$ since $r_{j+1}$ cannot cross the arc of $b_{j}$ again and the arc of $r_{j-1}$ is uncrossed due to invariant [3. We know that the special vertex of $g$ is $G$, otherwise the crossing between $b$ and $g$ would have been eliminated by Corollary 4 . This implies that $r_{j+1}$ must be incident to $G$ since the edge $g$ is crossed by $r_{j+1}$ if $y$ lies on $g$. Thus, the other endpoint of $r_{j+1}$, which is the endpoint of arc $r_{j+1}^{i}$, must be $G$. For arc $r_{j+1}^{i}$ to end in $G$, it must cross the curve $\alpha_{j}$. The arc $r_{j+1}^{i}$ cannot cross the arc of $r_{j-1}$ along $\alpha_{j}$ since the arc of $r_{j-1}$ along $\alpha_{j}$ is uncrossed by invariant 13 , and it cannot cross the arc of $b_{j}$ nor $g$ again. Hence, the crossing $y$ is not on the curve $\alpha_{j}$, which implies that $y$ lies in the interior of $f_{j}$. However, the interior of $f_{j}$ contains only $b$ and possibly $r_{0}$ due to invariant I5.

Edge $r_{j+1}$ cannot be incident to $R$, since otherwise $r_{j+1}$ would be an edge that is parallel to $g$. Therefore $r_{j+1}$ cannot cross $r_{0}$ whose special vertex is $R$.

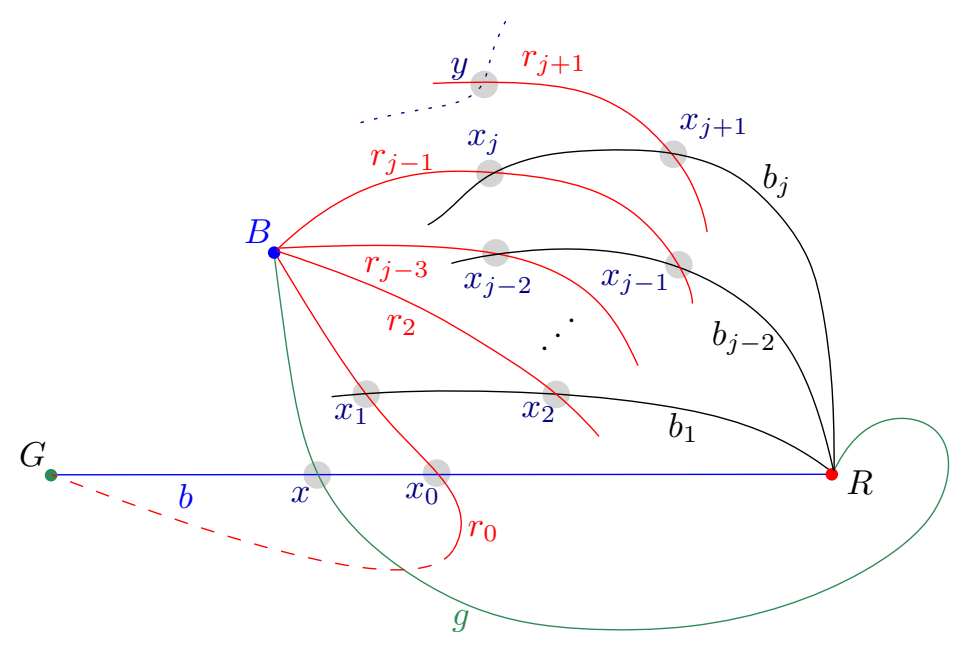

Fig. 13. Illustration of the case that $r_{j+1}$ is crossed between $B$ and $x_{j+1}$. 
This proves the proposition that the arc of $r_{j+1}$ between $x_{j+1}$ and $B$ is uncrossed in the drawing $\Gamma_{j+1}$, unless $j+1=k$.

Assume $r_{j+1} \neq q_{k}$. Then the arc of $r_{j+1}$ between $x_{j+1}$ and $B$ is uncrossed in the drawing $\Gamma_{j+1}$. Further, the arc of $b_{j}$ between $x_{j}$ and $R$ was uncrossed in $\Gamma_{j}$ by invariant I6. Since $r_{j+1}$ is the only new edge introduced for $\Gamma_{j+1}$, the arcs of $b_{j}$ between $x_{j}$ and $x_{j+1}$ as well as between $x_{j+1}$ and $R$ are uncrossed. The latter can be used in conjunction with the above proposition to conclude, that indeed, there is a face $f_{j+1}$ admitting invariant I5. To see this, note that $r_{j+1}$ cannot cross $g$, since it is not incident to $G$. Therefore, no additional edges cross $g$ when extending the drawing from $\Gamma_{j}$ to $\Gamma_{j+1}$. Invariant I5 can be combined with the fact that the arc of $r_{j-1}$ from $B$ to $x_{j}$ is uncrossed by invariant 13 to conclude that the triangular region $f_{j} \backslash f_{j+1}$ is empty and invariant $I 6$ is established. If the arc of $r_{j+1}$ between $x_{j+1}$ and $B$ is uncrossed, then it does not intersect $b$. On the other hand, we have already proved that the arc of $r_{j+1}$ that enters $f_{j} \backslash f_{j+1}$ at $x_{j+1}$ cannot leave this region, and $f_{j} \backslash f_{j+1}$ does not contain any part of $b$ by invariant I6. This finally proves invariant I4 and concludes the proof of the lemma in the case where $q_{j}=b_{j}$.

\section{G Proof of Theorem 1}

Proof (of Theorem 1). Let $\Gamma$ be a non-simple fan-planar drawing. By Corollary 4 we can find a fan-planar redrawing $\Gamma^{\prime}$ of $\Gamma$ such that

- no two edges cross more than once in $\Gamma^{\prime}$,

- if two adjacent edges cross in $\Gamma^{\prime}$, then their common endpoint is not the special vertex of either of the two edges; , and

$-\Gamma^{\prime}$ does not have more crossings than $\Gamma$.

If $\Gamma^{\prime}$ still contains adjacent crossings, we can apply Lemma 4 to obtain a fanplanar redrawing of $\Gamma^{\prime}$ with fewer crossings. Since the number of crossings is finite, we can iterate this procedure to eventually obtain a simple fan-planar drawing. 